\title{
Surfactant sputtering
}

\author{
Hans Hofsäss • Kun Zhang
}

Received: 8 April 2008 / Accepted: 15 May 2008 / Published online: 28 May 2008

(C) The Author(s) 2008

\begin{abstract}
Sputter erosion of materials is among the most important techniques for fabricating advanced thin film coatings. Sputter processes are also of considerable relevance for surface polishing down to an atomic scale, nanostructuring of surfaces as dot and ripple patterns and micromachining of materials using focused ion beams or reactive ion etching. We present a new, versatile sputter technique utilizing the steady state coverage of a substrate surface with up to $10^{16} \mathrm{~cm}^{-2}$ of foreign or self atoms simultaneously during sputter erosion by combined ion irradiation and atom deposition. These surfactant atoms (surface active agents) strongly modify the substrate sputter yield on atomic to macroscopic length scales. Depending on the surfactantsubstrate combination, the novel technique allows enhanced smoothing of surfaces, the generation of novel surface patterns and nanostructures and the controlled shaping of surfaces on the nanometer scale. We present selected examples of surface morphology evolution, smoothing of surfaces and shaping of surfaces to demonstrate the capabilities of the new surfactant sputtering technique.
\end{abstract}

PACS 79.20.rf $\cdot 81.40 .-\mathrm{z} \cdot 81.65 .-\mathrm{b} \cdot 61.80 . \mathrm{Jh} \cdot 68.55 .-\mathrm{a}$

\section{Introduction}

Sputtering of surfaces by particle bombardment has found manifold applications in technology and research. Many publications document the progress in sputter technology

H. Hofsäss $(\varangle) \cdot K$. Zhang

II. Physikalisches Institut, Universität Göttingen, Friedrich-Hund-Platz 1, 37077 Göttingen, Germany e-mail: hans.hofsaess@phys.uni-goettingen.de during the last four decades, regarding applications, development of new sputter techniques, theoretical understanding, modeling, and computer simulation [1-4]. Modifications of sputtering techniques were introduced, such as chemical sputtering or reactive sputtering [5], cluster beam sputtering [6], and metal assisted sputtering for secondary ion mass spectrometry (MetA-SIMS) [7]. Sputter processes are of considerable importance for fabricating thin film coatings using techniques like ion assisted deposition, ionized physical vapor deposition (PVD), and plasma based deposition techniques $[8,9]$. Several PVD techniques utilize either multiple ion beams or multiple sputter targets, such as dual ion beam sputtering (DIBS) [10] or multiple source sputter deposition [11-14]. Dual ion beam deposition uses either sequential or simultaneous deposition of film species supplied from different ion sources, such as mass selected ion beam deposition $[15,16]$ or filtered cathodic arc deposition using multiple cathodes [17]. Since these methods are devoted to thin film synthesis, the deposition rate always considerably exceeds the sputter rate. The ion assisted synthesis of cubic boron nitride (c-BN) films is one of the few cases where film growth takes place near the re-sputtering limit $[18,19]$. At the re-sputtering limit, the sputter and deposition rates balance and there is no net film deposition. The situation where sputtering outbalances deposition in ion assisted deposition techniques is of no interest for film growth and therefore it was paid no attention so far.

Shaping surfaces with a precision on the nanometer scale or even atomic scale is becoming more and more important in advanced materials synthesis and nanotechnology. Shaping includes smoothing [20, 21], nanopattern formation [22, 23, 41] and micro- and nano-machining of 2Dand 3D-structures using focused ion beams [24]. Here, sputter processes provide unique possibilities regarding resolution, precision, process control, and process scalability. Re- 
deposition of sputtered material is also an issue in manufacturing of nanoscale structures [25-28]. However, in most cases re-deposition of sputtered material is seen as a contamination with unwanted species and many publications deal with procedures to avoid re-deposition [29].

Deposition during sputtering was applied by Berg et al. to explain sputter yield amplification [30-32]. During ion assisted deposition, the sputter yield of a thin layer of low mass atoms on a high mass matrix is enhanced if the thickness of the layer is below a few nm. This effect is caused by differences in the collision cascades in the layers. Berg et al. come to the conclusion, that "by deliberately adding impurity atoms to the surface of a substrate, it is possible to influence the collision cascade by an incoming ion in such a way as to promote excess sputtering from the surface" [32]. However, SRIM [33] simulations and our results presented below show no sputter yield amplification in most cases, particularly in the case of inclined incidence of the eroding ion beam onto the substrate. In contrast, a surface coverage of a substrate by co-deposition of atoms strongly reduces the substrate sputtering yield.

Here we present "surfactant sputtering" as a novel sputter technique for the erosion of surfaces which utilizes the steady state coverage of the surface with typically $<10^{16} \mathrm{~cm}^{-2}$ of foreign or self atoms simultaneously during ion beam sputtering [34]. The coverage with these atoms can be achieved by combined particle irradiation of a substrate, i.e. ion irradiation and simultaneous sputter deposition, evaporation or low energy ion deposition, or laser ablation. These foreign- or self atoms act as surface active agents (surfactants) allowing one to manipulate the sputtering yield of substrate atoms in manifold ways and on length scales from the nanometer range to macroscopic dimensions. The term surfactant is a blend of "surface active agent" and is used, for example, to describe epitaxial growth of semiconductor or metal films where surfactant atoms or molecules form a surface layer promoting ideal epitaxial growth $[35,36]$. With the help of surfactants we can adjust the sputtering yield from the value of the pure surface down to zero. Moreover, depending on alloy formation with substrate atoms, surface segregation, island formation, cluster formation, or attachment to surface defects, the surface coverage with surfactant atoms may be quite inhomogeneous and may lead to inhomogeneous sputtering on a nanometer scale.

\section{Experimental}

For surfactant sputtering the surfactant deposition rate onto the substrate is usually small enough so that a net erosion of the substrate including the surfactant atoms takes place. The situation is therefore comparable to ion assisted deposition (IBAD) operated beyond the re-sputtering limit. The technique could be also named 'particle beam assisted sputtering (PBAS)', in analogy to IBAD.

An obvious setup for surfactant sputtering experiments would be quite similar to an IBAD setup using two ion sources, a sputter target and a substrate. Preferably, the sputter target and also the ion beam irradiating the substrate are alternatingly blocked by shutters to prevent a contamination of the sputter target due to re-deposition of atoms sputtered off the substrate. Several other experimental setups, e.g., using rotating substrates and rotating sputter targets, may also be suitable.

For the present studies we have chosen a setup consisting of a single broad beam ion source and a geometrical arrangement of substrate and sputter target so that both are exposed to this ion beam (Fig. 1). The source of surfactant atoms is a sputter target positioned behind the substrate (relative to the ion beam direction) but inclined to the substrate, so that a fraction of atoms sputtered off the target are directly deposited onto the substrate. Angle $\alpha$ adjusts the overall substrate sputter yield and also determines the formation of ripple patterns and similar nanostructures on the substrate surface. Angle $\beta$ determines the gradient of surfactant coverage across the substrate. Substrate positions given below refer to the scale shown in Fig. 1. Typically the steady-state coverage of surfactants increases from position $0 \mathrm{~mm}$ to position $7 \mathrm{~mm}$.

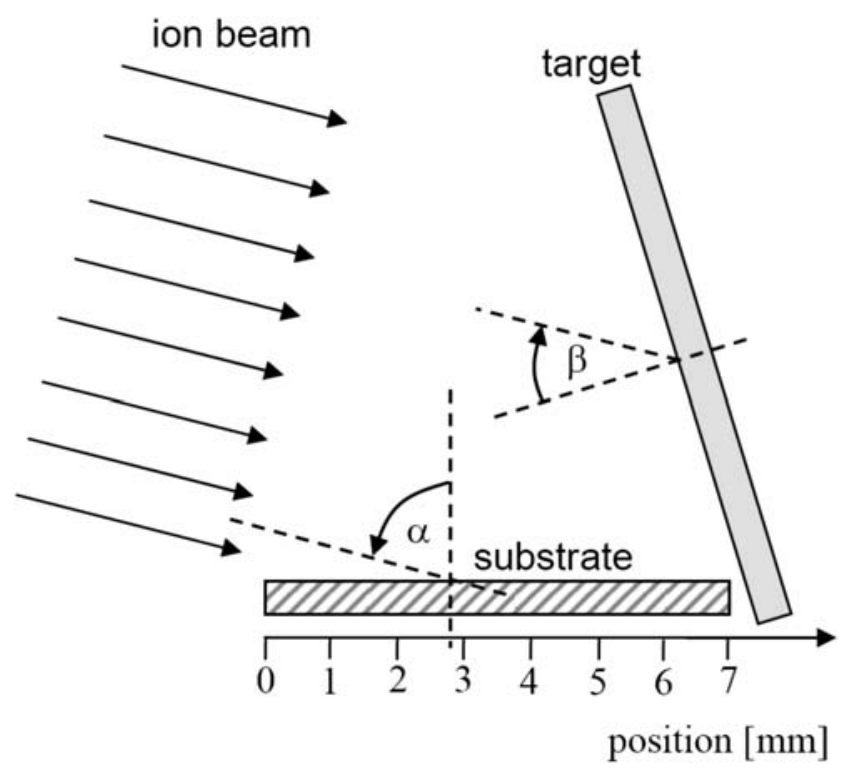

Fig. 1 Schematic experimental setup used for surfactant sputtering. A broad and homogeneous ion beam is incident on a substrate to be sputtered at an incidence angle $\alpha$ with respect to the substrate surface normal. The ion beam also irradiates a sputter target at an incidence angle $\beta$ with respect to the target surface normal. The target is positioned in such a way, that atoms sputtered off the target can be directly deposited onto the substrate, resulting in lateral inhomogeneous steady state coverage of the surface with surfactant atoms. Different substrate positions indicated in the text refer to the shown scale 
The advantage of this setup is the possibility to vary the surfactant coverage across the substrate area, providing an easy way to study erosion effects as a function of surfactant coverage. The disadvantage is the deposition of atoms sputtered off the substrate onto the sputter target. However, for the chosen substrate-target geometries (angle $\beta=0^{\circ}$ and angle $\alpha=70^{\circ}-80^{\circ}$ ) the approximate $\cos \Theta$ angular distribution of sputtered atoms ensures that the deposition rate on the target is always less than the sputter rate from the target. Thus, we only generate steady-state coverage on the surface of the sputter target, which in turn influences the steady-state surfactant coverage on the substrate. The steady-state coverage of the sputter target does not pose a problem for the present studies, since we are at first interested in generating a certain steady-state surfactant coverage with a gradient across the substrate, increasing from position $0 \mathrm{~mm}$ to position $7 \mathrm{~mm}$. The amount of coverage can be directly determined later on, e.g., by Rutherford backscattering spectroscopy (see Example 4) or indirectly by measuring the sputter depth using profilometry. It is clear, however, that deposition onto the target has to be taken into account for a quantitative prediction of the steady-state surfactant coverage for a given target-substrate geometry.

Sputter erosion studies presented here were carried out at room temperature using a $5 \mathrm{keV}$ mass selected Xe-ion beam, ion fluences up to $1.2 \times 10^{17} \mathrm{~cm}^{-2}$ and an incident angle $\alpha$ of $70^{\circ}$ or $80^{\circ}$. The pressure during erosion was $2 \times 10^{-6} \mathrm{~Pa}$. The ion flux was about $2 \mu \mathrm{A} / \mathrm{cm}^{2}$, so that heating of the samples by the ion beam is negligible. The ion beam was provided by a Colutron Model G2 mass selective ion beam system using a plasma ion source and a Wien-filter for mass selection [37]. The beam with diameter of few $\mathrm{mm}$ and beam divergence of less than $1^{\circ}$ was swept across the sample area using an electrostatic beam sweep system. The typical samples size was $10 \times 7 \mathrm{~mm}^{2}$ and the homogeneous ion beam cross section was about $1.2 \mathrm{~cm}^{2}$. As samples we used silicon, silicon with polymer resist surface patterns, thermally grown silicon dioxide on $\mathrm{Si}$, and metal films (Fe, $\mathrm{Ni}$ ) evaporated on silicon or glass substrates.

The substrates and the films, prior and after sputter erosion, were analyzed by atomic force microscopy and scanning electron microscopy. Rutherford backscattering spectroscopy (RBS) using a $900 \mathrm{keV} \mathrm{He}^{2+}$ beam was applied to determine the initial and final film thickness, the experimental sputtering yield and the sputter depth. The sputter depth of some samples was also analyzed using a profilometer.

\section{Results and discussion}

We have investigated surfactant sputtering for a variety of surfactant-substrate combinations, different angles of incidence of the ion beam and different inclination between sputter target and substrate. In the following we present selected examples demonstrating the diversity of novel surface morphologies which may occur and also the possibility of controlled shaping and smoothing of surfaces. Further results will be described in forthcoming publications. The chosen examples are:

(i) Sputter erosion of silicon with Si surfactants.

(ii) Sputter erosion of silicon with $\mathrm{Au}, \mathrm{Ag}$, and Pt surfactants.

(iii) Generation of thickness gradients in thermally grown $\mathrm{SiO}_{2}$ films on $\mathrm{Si}$ by sputter erosion with $\mathrm{Al}$ surfactants.

(iv) Generation of thickness gradients in $\mathrm{Fe}$ thin films by sputter erosion with $\mathrm{Cu}$ surfactants.

Example 1 In this reference experiment, Si substrates were sputter-eroded at an incidence angle of $\alpha=70^{\circ}$ and a fluence of $10^{17} \mathrm{Xe}^{+} / \mathrm{cm}^{2}$ and, in addition, $\mathrm{Si}$ was deposited from a sputter target inclined to the substrate so that the ion beam was incident parallel to the target surface normal $\left(\beta=0^{\circ}\right)$. The Si deposition flux was low enough to ensure net erosion of the substrate. Across the width of the substrate the deposition flux varied from a high value at position $7 \mathrm{~mm}$ to a low value at the position $0 \mathrm{~mm}$. We observe ripple patterns with wave vector parallel to the projected direction of the ion beam, wavelength of about $40 \mathrm{~nm}$ and $\mathrm{rms}$ roughness of $1.35 \mathrm{~nm}$, nearly independent of the steady state coverage with Si surfactants (Fig. 2, upper row). This is comparable to sputter erosion without surfactants [38]. However, the effective sputtering yield decreases from the position $0 \mathrm{~mm}$ towards position $7 \mathrm{~mm}$. The gradient in sputter depth across the sample was about $80 \mathrm{~nm} / \mathrm{cm}$ per $10^{17} \mathrm{ion} / \mathrm{cm}^{2}$. The experiment shows that the ripple pattern formation is not influenced by simultaneous deposition of $\mathrm{Si}$, but the effective substrate sputter yield can be adjusted from the bulk value (without surfactants) down to zero. Even a negative effective sputter yield (growth condition) is possible. In principle, it is possible to generate a nanostructured rippled surface by surfactant ion beam erosion without net erosion of material. This could be interesting for generating rippled surfaces on thin film coatings without modifying the film thickness.

Example 2 We now use $\mathrm{Au}, \mathrm{Ag}$, or Pt foils as sputter targets and the same irradiation geometry as in Example 1. Again, the deposition flux onto the Si substrate is low enough to ensure net erosion of the substrate. In this situation, we obtain a small steady state surface coverage of the substrate with metal atoms, which modifies the ripple pattern formation dramatically.

The corresponding AFM pictures for Si erosion using $\mathrm{Au}$ surfactants and an ion fluence of $10^{17} \mathrm{Xe} / \mathrm{cm}^{2}$ are displayed in Fig. 2 (middle and lower row). For position $2 \mathrm{~mm}$ the ripple pattern coarsens and the wavelength increases to about $50 \mathrm{~nm}$ in comparison to sputtering with $\mathrm{Si}$ surfactants. There 
Fig. 2 AFM pictures of $\mathrm{Si}$ surfaces eroded with $1 \times$ $10^{17} \mathrm{Xe} / \mathrm{cm}^{2}$ at $5 \mathrm{keV}$ and $\alpha=70^{\circ}$ with $\mathrm{Si}$ and $\mathrm{Au}$ surfactants. The arrow indicates the projected ion beam direction. Upper row: Ripple patterns for Si surfactants. Middle row and lower row: Ripple patterns for $\mathrm{Au}$ surfactants. The Au coverage increases from position $2 \mathrm{~mm}$ to position $6 \mathrm{~mm}$ and the $\mathrm{rms}$ roughness strongly decreases, resulting in a flat surface at position $6 \mathrm{~mm}$. Lower row, right: rms roughness for different positions across the sample, showing the drastic decrease of roughness with increasing coverage with $\mathrm{Au}$ surfactants

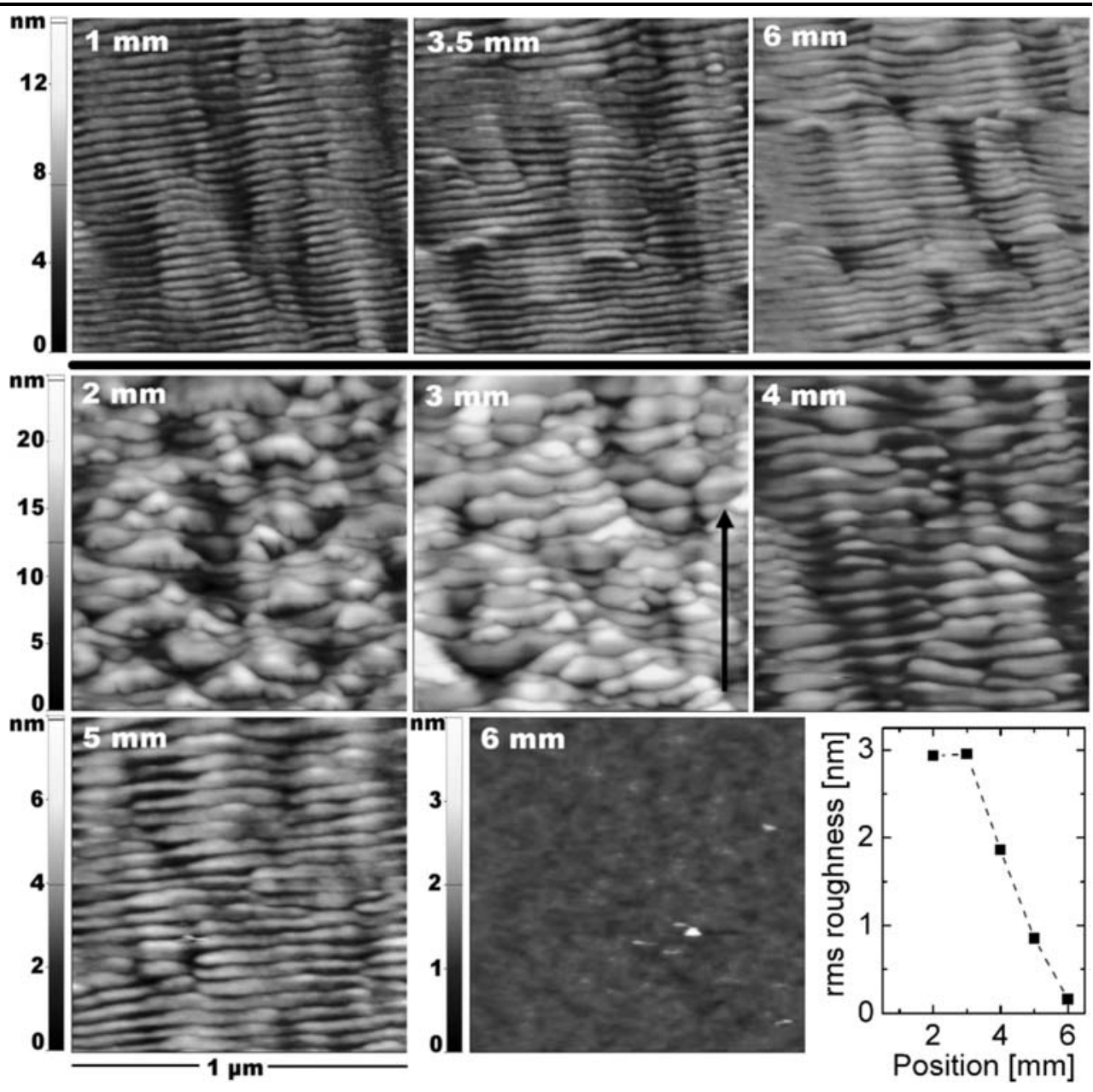

may be indications for $\mathrm{Au}$ or $\mathrm{Au}_{x} \mathrm{Si}$ cluster formation at the surface. At intermediate positions $(3-4 \mathrm{~mm})$, the patterns become smoother with shorter wavelength and eventually at position $6 \mathrm{~mm}$ the surface becomes extremely smooth (rms roughness below $0.2 \mathrm{~nm}$ ). RBS analyses reveal a surface coverage with $\mathrm{Au}$ increasing from $2 \times 10^{15} \mathrm{Au} / \mathrm{cm}^{2}$ at position $1 \mathrm{~mm}$ to $1 \times 10^{16} \mathrm{Au} / \mathrm{cm}^{2}$ at position $6 \mathrm{~mm}$, corresponding to an effective Au film thickness between 0.4 and $1.7 \mathrm{~nm}$. Surprisingly, profilometer measurements of the sputter depth indicate only a slightly smaller sputtering yield compared to pure Si and only a weak variation of the sputtering yield at different sample positions. This is an indication for the existence of a silicon surface layer and the formation of a buried mixed $\mathrm{Au} / \mathrm{Si}$ layer in the surface near region. The buried layer is most likely formed due to recoil implantation of Au below the Si surface. We speculate that the smoothing effect is due to ion-induced plastic flow within the buried layer and ion-induced athermal formation of $\mathrm{Au}_{x} \mathrm{Si}$ in the subsurface region approaching the eutectic composition $\mathrm{Au}_{4} \mathrm{Si}$ (with lowest melting temperature) at position $6 \mathrm{~mm}$. The ion-induced formation of $\mathrm{Au}-\mathrm{Si}$ bonds is supported by the work of Pászti et al. [39].
Surfactant sputtering of Si using $\mathrm{Ag}$ and $\mathrm{Pt}$ foils as sputter target and an ion fluence of $3 \times 10^{16} \mathrm{Xe} / \mathrm{cm}^{2}$ leads to surface morphologies as shown in the SEM pictures displayed in Fig. 3. In the case of $\mathrm{Ag}$, the ripple pattern at position $1 \mathrm{~mm}$ is quite similar to that of pure Si. For positions towards the Ag sputter target, the pattern coarsens and the wavelength increases above $50 \mathrm{~nm}$. AFM als reveals an increase of the rms roughness above $4 \mathrm{~nm}$. The SEM pictures in Fig. 3 show an increasing amount of small $\mathrm{Ag}$ clusters on the flat plateaus of surface. The size of these clusters is $10 \mathrm{~nm}$ and less, in agreement with TEM studies showing that low energy ion irradiation of Ag cluster films on Si reduces the average cluster size below $10 \mathrm{~nm}$ [39]. We explain the coarsening of the ripple pattern in our case by dynamic clustering of $\mathrm{Ag}$ on the surface reaching a steady state average cluster size and cluster density. Each cluster influences the erosion of the Si substrate by shadowing a fraction of the surface, thus preventing erosion within the shaded substrate area. Also shown in Fig. 3 are patterns obtained after sputtering of Si using Pt surfactants. In this case the ripple patterns coarsen dramatically compared to erosion of pure $\mathrm{Si}$ and also with increasing Pt surfactant coverage. It is most 


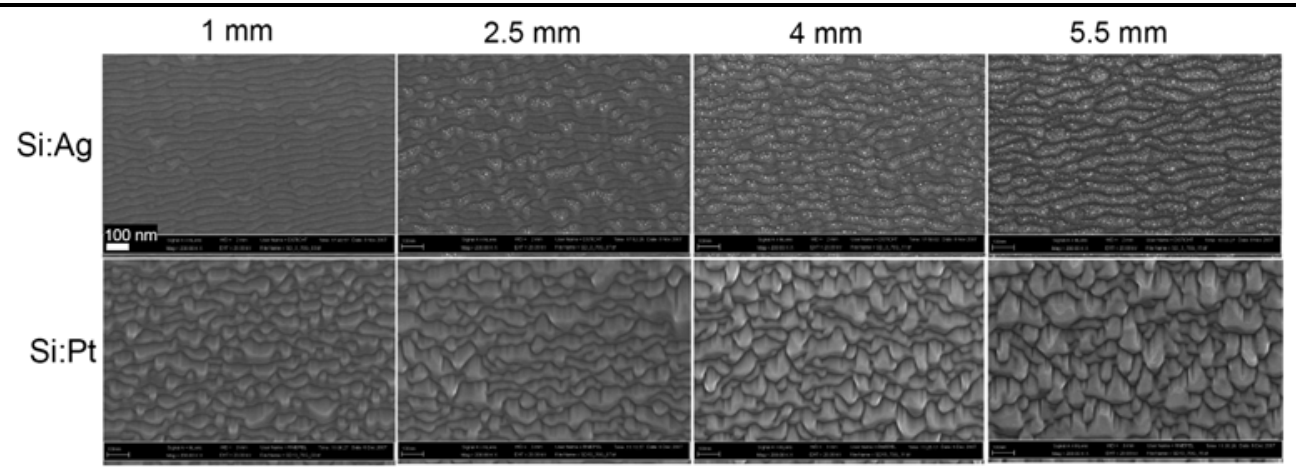

Fig. 3 SEM pictures of Si surfaces eroded with $5 \mathrm{keV} \mathrm{Xe} \mathrm{@} 70^{\circ}$ and $3 \times 10^{16} \mathrm{~cm}^{-2}$ with Ag surfactants (top row) and Pt surfactants (bottom row). The indicated positions refer to the scale shown in Fig. 1. The ripple pattern and ripple wavelength at different positions on the

likely that a thin platinum silicide surface layer is formed which drastically modifies the pattern formation processes.

Example 3 This simple experiment demonstrates the controlled preparation of thickness gradients in thin films using surfactant sputtering. A Si substrate was thermally oxidized to an oxide thickness of about $450 \mathrm{~nm}$ and subsequently sputter-eroded at an incidence angle of $\alpha=80^{\circ}$ and a fluence of $3 \times 10^{16} \mathrm{Xe} / \mathrm{cm}^{2}$. As sputter target we used $\mathrm{Al}$ inclined at $\beta=30^{\circ}$ to the ion beam. Prior to erosion, part of the substrate was covered with a piece of Si to perform profilometer measurements later on. The optical micrograph of the sample taken after sputter erosion clearly shows a pronounced thickness gradient (Fig. 4). Interference colors as well as profilometer data reveal the increasing residual thickness of the $\mathrm{SiO}_{2}$ layer from about $310 \mathrm{~nm}$ (position $0 \mathrm{~mm}$, bottom of Fig. 4) to $430 \mathrm{~nm}$ (position $7 \mathrm{~mm}$, top of Fig. 4). Thus, the measured effective $\mathrm{SiO}_{2}$ sputtering yield is about 32 atoms/ion (close to the sputter yield without surfactants and comparable to the calculated yield 29 atoms/ion using TRIDYN [40]) at the bottom and decreases to about 4.6 atoms/ion at the top, corresponding to a slope of about $60 \mathrm{~nm} / \mathrm{cm}$ per $10^{16} \mathrm{ion} / \mathrm{cm}^{2}$. Further results regarding the preparation of wave guide structures or the fabrication of trenches with depth gradients will be presented in a forthcoming publication.

Example 4 During sputtering of initially $140 \mathrm{~nm}$ thick Fe thin films on glass using $5 \mathrm{keV} \mathrm{Xe}$ ions at $\alpha=80^{\circ}$ the films were exposed to a flux of $\mathrm{Cu}$ atoms, originating from a $\mathrm{Cu}$ sputter target with surface normal parallel to the ion beam $\left(\beta=0^{\circ}\right)$. After sputter erosion, a fluence dependent thickness gradient was measured using cross sectional SEM (Fig. 5). For sputtering of pure Fe we expect a sputter yield of $Y_{\mathrm{Fe}}^{\text {bulk }} \approx 15.3$ atoms/ion (from SRIM [33]) and a corresponding sputter depth of about $220 \mathrm{~nm}$ at fluence substrate is strongly influenced by the surfactants. Ag nanoparticles of size $10 \mathrm{~nm}$ or less can be seen on the top of the flat ripple plateaus. In the case of Pt surfactants, a platinum silicide surface layer is most probably formed

$1.2 \times 10^{17} \mathrm{ion} / \mathrm{cm}^{2}$. Instead, the effective sputter yield varies between 12 and 3 atoms/ion across the sample. The $\mathrm{Cu}-\mathrm{Fe}$ system is immiscible and we expect that surfactant sputtering with $\mathrm{Cu}$ leads to a steady state $\mathrm{Cu}$ surface coverage which reduces the $\mathrm{Fe}$ sputtering yield. AFM analyses of ripple patterns measured after erosion at different sample positions did not show $\mathrm{Cu}$ cluster formation [41]. This indicates a rather homogeneous steady state surface coverage. At the end of the ion irradiation, the surface coverage in dynamic equilibrium is frozen in and remains as an ultra-thin surface layer. RBS analyses reveal a layer thickness varying from $0.05 \mathrm{~nm}$ and $0.4 \mathrm{~nm}$ across the samples, independent of the ion fluence. This independence is expected for a steady-state surface coverage. Furthermore, the example demonstrates that surfactant sputtering may also be used to generate surfaces covered with ultra-thin films.

In the following, we present a model to describe the effect of surfactant atoms for a representative non-miscible system like $\mathrm{Cu}$ on Fe. From SRIM [33] simulations we calculate the sputtering yield of $\mathrm{Cu}$ and $\mathrm{Fe}$ for a homogeneous thin $\mathrm{Cu}$ surfactant layer on a $\mathrm{Fe}$ substrate with variable layer area density $\sigma$ up to $10^{16}$ atoms $/ \mathrm{cm}^{2}$, corresponding to a layer thickness up to $1 \mathrm{~nm}$. We find an exponential decrease of the Fe sputtering yield with characteristic coverage $\sigma_{1}$ and a corresponding exponential increase towards saturation of the $\mathrm{Cu}$ sputtering yield with increasing $\mathrm{Cu}$ layer thickness with a characteristic coverage $\sigma_{0}$. For $5 \mathrm{keV}$ Xe incident at $\alpha=80^{\circ}$ the sputter yields of $\mathrm{Cu}$ surfactants $Y_{\mathrm{S}}$ and $\mathrm{Fe}$ substrate $Y_{\text {Substrate }}$ are determined from SRIM [33] simulations as

$$
\begin{aligned}
& Y_{\mathrm{S}}(\sigma)=Y_{\mathrm{S}}^{\text {bulk }}\left(1-\mathrm{e}^{-\sigma / \sigma_{0}}\right) \\
& \quad \text { with } \sigma_{0}=1.22 \times 10^{15} \mathrm{~cm}^{-2} \text { and } Y_{\mathrm{S}}^{\text {bulk }} \approx 20.5 \frac{\text { atoms }}{\text { ion }} \\
& \quad \text { for } \mathrm{S}=\mathrm{Cu},
\end{aligned}
$$




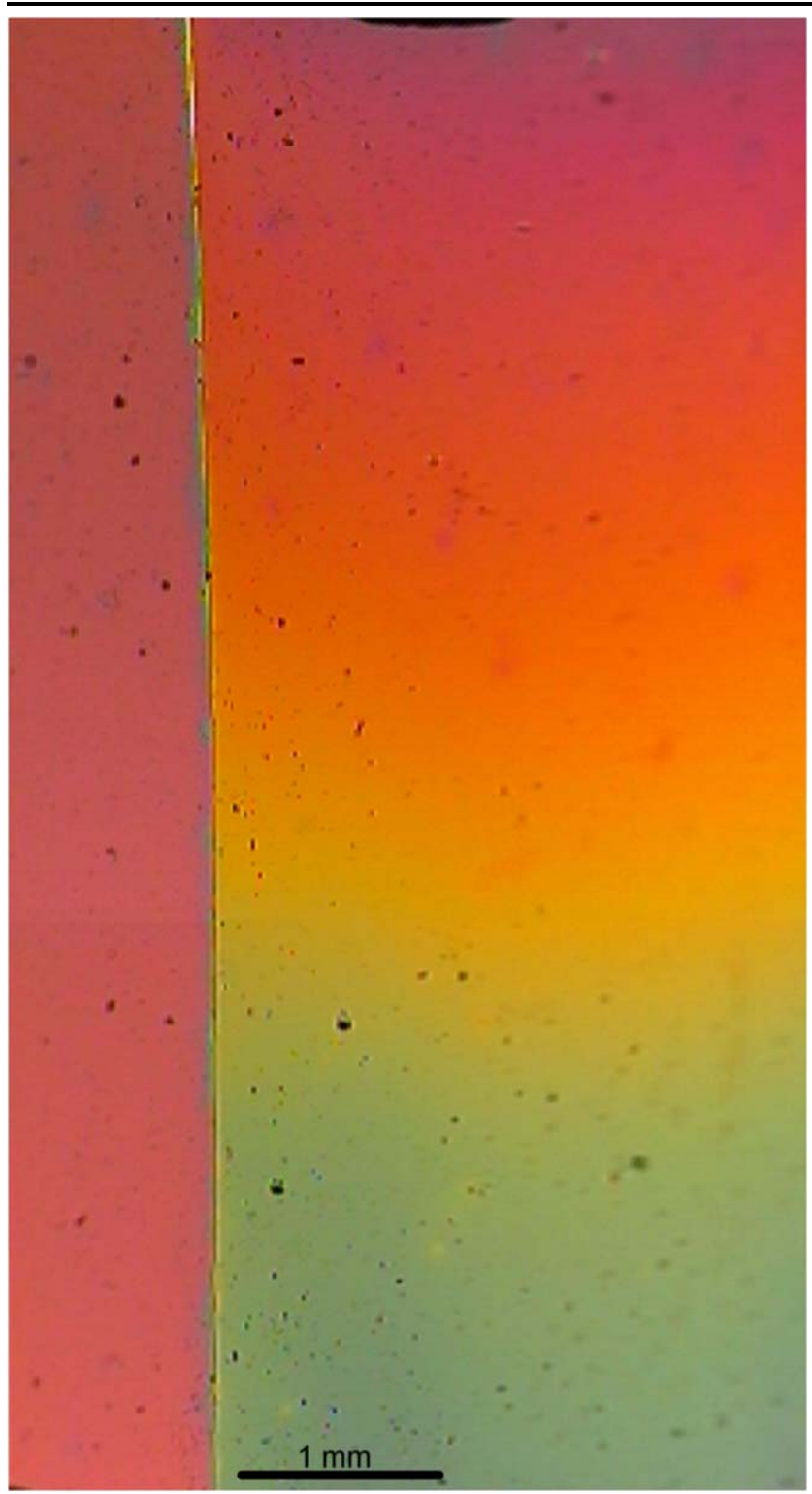

Fig. 4 Photograph of a $\mathrm{SiO}_{2}-\mathrm{Si}$ sample after surfactant sputtering using $\mathrm{Xe}$ ions and $\mathrm{Al}$ surfactants. Ion-irradiation was done using $5 \mathrm{keV}$ Xe with $3 \times 10^{16} / \mathrm{cm}^{2}$ incident at $\alpha=80^{\circ}$ from below. The Al sputtering target was positioned above the upper boundary of the sample inclined at $\beta=30^{\circ}$ to the ion beam direction. The left part of the sample was not exposed to the ion beam and has an oxide thickness of $450 \mathrm{~nm}$. The right part was exposed to the ion beam and simultaneously covered with Al surfactants during erosion with area density increasing from the bottom to the top. The interference colors from blue-green (bottom) over yellow to magenta indicate the increasing residual thickness of the $\mathrm{SiO}_{2}$ layer from 310 to $430 \mathrm{~nm}$

$$
\begin{aligned}
& Y_{\text {Susbtrate }}(\sigma)=Y_{\text {Substrate }}^{\text {bulk }} \mathrm{e}^{-\sigma / \sigma_{1}} \\
& \text { with } \sigma_{1}=1.11 \times 10^{15} \mathrm{~cm}^{-2} \text { and } Y_{\text {Substrate }}^{\text {bulk }} \approx 15.3 \frac{\text { atoms }}{\text { ion }}
\end{aligned}
$$$$
\text { for Substrate }=\text { Fe }
$$

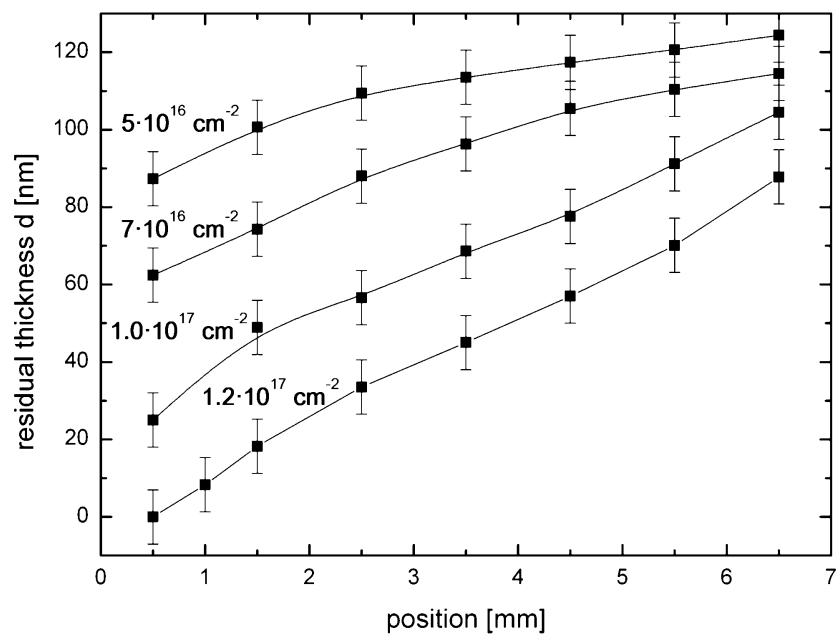

Fig. 5 RBS analysis of the residual film thickness $d$ after erosion of initially $140 \mathrm{~nm}$ thick Fe films, measured at different positions across the films. Surfactant sputtering was done at different ion fluences with $5 \mathrm{keV} \mathrm{Xe}$ ions at $\alpha=80^{\circ}$ incidence angle and $\mathrm{Cu}$ surfactants from a $\mathrm{Cu}$ sputter target inclined at $\beta=0^{\circ}$. The average sputter depth is from top to bottom 30, 47, 73, and $94 \mathrm{~nm}$, which gives an average sputter yield of $Y=6.0(7)$. The local sputter yield varies by a factor of $0.5-2$ across the sample (from [42])

Using this functional dependence and a constant positive deposition flux $j_{\mathrm{D}}$ of surfactant atoms and ion flux $j_{\text {Ion }}$, we obtain a rate equation for the surfactant area density $\sigma(t)$, given by

$\frac{d \sigma}{d t}=-j_{\mathrm{Ion}} Y_{\mathrm{S}}^{\mathrm{bulk}}\left(1-\mathrm{e}^{-\frac{\sigma}{\sigma_{0}}}\right)+j_{\mathrm{D}}$,

with bulk surfactant sputtering yield $Y_{\mathrm{S}}^{\text {bulk }}$. The solution of (2) is

$\sigma(t)=\sigma_{0} \ln \left(\frac{a \mathrm{e}^{t(a-b)}-b}{(a-b)}\right)$ for $a \neq b$,

$\sigma(t)=\sigma_{0} \ln (a t+1) \quad$ for $a=b$

with $a=j_{\mathrm{D}} / \sigma_{0}$ and $b=j_{\mathrm{Ion}} Y_{\mathrm{S}}^{\text {bulk }} / \sigma_{0}$ and $\frac{a}{b}=\frac{j_{\mathrm{D}}}{j_{\mathrm{Ion}} Y_{\mathrm{S}}^{\text {bulk }}}$.

For $a<b$, the substrate sputtering yield is given by inserting (3) into (1b):

$$
\begin{aligned}
Y_{\text {Substrate }} & =Y_{\text {Substrate }}^{\text {bulk }}\left(1-\frac{a}{b}\right)^{\sigma_{0} / \sigma_{1}} \\
& =Y_{\text {Substrate }}^{\text {bulk }}\left(1-\frac{j_{\mathrm{D}}}{j_{\text {Ion }} Y_{\mathrm{S}}^{\text {bulk }}}\right)^{\sigma_{0} / \sigma_{1}}
\end{aligned}
$$

We see from (4) that the reduction of the substrate sputtering yield is essentially determined by the ratio of surfactant deposition flux $j_{\mathrm{D}}$ and maximum surfactant erosion flux $j_{\mathrm{I}} Y_{\mathrm{S}}^{\text {bulk }}$. Since in our example $(\mathrm{Cu}$ on $\mathrm{Fe}) \sigma_{0} \approx \sigma_{1}$, the target sputter yield decreases almost linearly with increasing surfactant deposition flux $j_{\mathrm{D}}$. 


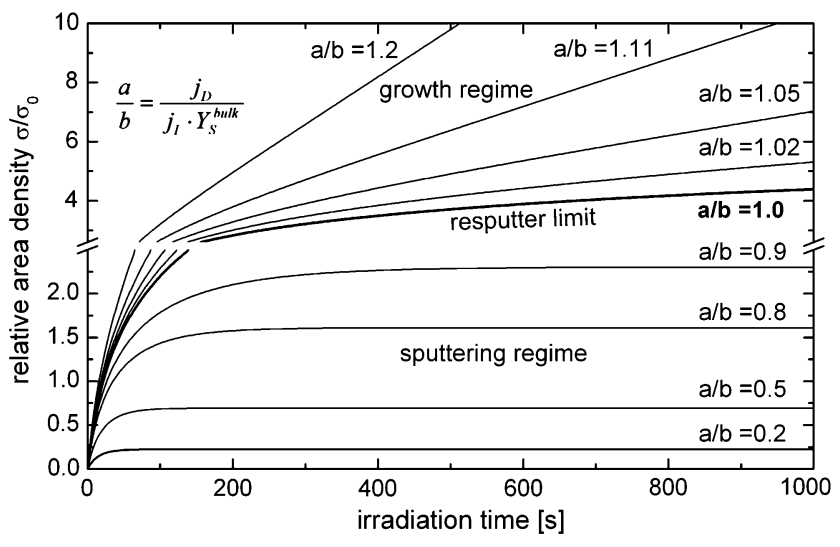

Fig. 6 Relative area density of surfactant atoms as a function of irradiation time and ratio $a / b$ calculated from (3). Surfactant sputtering utilizes the saturation surface coverage within the sputtering regime where $a / b<1$

In Fig. 6 we have plotted $\sigma(t)$ from (3) for various ratios $a / b$. We can clearly distinguish the growth regime with linear increase of film thickness for large $t$. In the sputtering regime $a<b$, which is the regime beyond the re-sputtering limit in ion assisted deposition, the surfactant area density reaches a saturation value given by

$\sigma^{\text {sat }}=\sigma_{0} \ln \left(\frac{1}{1-a / b}\right)$.

For $a / b$ varying between 0.01 and 0.99 and for $\sigma_{0} \approx$ $1.22 \times 10^{15} \mathrm{~cm}^{-2}(\mathrm{Cu}$ on $\mathrm{Fe})$ the saturation coverage $\sigma^{\text {sat }}$ varies according to (5) from $1.23 \times 10^{13}$ to $5.6 \times 10^{15} \mathrm{~cm}^{-2}$, and the target sputtering yield $Y_{\text {Substrate }}$ varies according to (4) from $0.99 \cdot Y_{\text {Substrate }}^{\text {bulk }}$ to $0.0095 \cdot Y_{\text {Substrate }}^{\text {bulk }}$.

This behavior is observed in our experiments described in Example 4 and shown in Fig. 5. In this example we obtain a variation of the effective Fe sputter yield between 12 and 3 atoms/ion, corresponding to a ratio $0.19 \leq a / b \leq 0.77$ as calculated from (4). This corresponds to a saturation area density of $\mathrm{Cu}$ varying between $2.6 \times 10^{14}$ and $1.8 \times$ $10^{15} \mathrm{~cm}^{-2}$, or a Cu layer thickness between 0.03 and $0.2 \mathrm{~nm}$. The somewhat higher value determined from RBS is possibly due to a small amount of dispersed $\mathrm{Cu}$ atoms recoil implanted in to the subsurface region of the Fe film.

\section{Conclusions}

We introduce "surfactant sputtering" as a novel and versatile sputtering technique utilizing a steady-state surface coverage of the surface with suited surfactant atoms simultaneously during ion beam erosion. In general, surfactant atoms cause a pronounced reduction of the substrate sputtering yield. Furthermore, depending on the behavior of the surfactant atoms (clustering, island formation, attachment to surface defects and grain boundaries, etc.) and their chemical interaction with the substrate (alloy and compound formation) the erosion process becomes inhomogeneous on a local atomic scale up to macroscopic length scales. As a result, the pattern formation during sputter erosion is strongly influenced by surfactants leading to a wide variety of new surface morphologies on the nm scale. Furthermore, surfactants can be used to significantly enhance the surface smoothness and suppress ripple formation at glancing angle ion beam erosion, as shown for Au surfactants on Si. Thin films with thickness gradients and trenches with adjustable depth gradients can easily be fabricated using surfactant sputtering with gradients as low as $10^{-6}(10 \mathrm{~nm}$ over $1 \mathrm{~cm})$. Possible applications may be waveguide structures and other passive optical devices with thickness gradient structures. As shown for $\mathrm{Cu}$ surfactants on $\mathrm{Fe}$, the technique may also be used to grow ultra-thin films. Many more applications of surfactant sputtering for surface treatment and surface processing still have to be explored. We have shown for $\mathrm{Cu}$ on $\mathrm{Fe}$ that rate equations are in some cases suitable to describe the steady state surface coverage and the resulting effective sputter yields. Open questions are related to the interaction of surfactant atoms with the substrate under the influence of the ion beam; in particular the formation of compounds and alloys, and the mechanism of ripple formation under these conditions. A quantitative description of the steadystate coverage for a given substrate-target geometry, taking into account the angular distribution of sputtered atoms and the effect of re-deposition onto the target is currently under development.

Acknowledgements We thank Prof. Johann Krauser and Daniel Stichtenoth for their help with AFM analyses and SEM analyses and Prof. Klaus-Peter Lieb and Prof. Krish Bharuth-Ram for valuable discussions when preparing the manuscript. Financial support by the German Research Society within the collaborative research center SFB 602 project B2 is gratefully acknowledged.

Open Access This article is distributed under the terms of the Creative Commons Attribution Noncommercial License which permits any noncommercial use, distribution, and reproduction in any medium, provided the original author(s) and source are credited.

\section{References}

1. R. Behrisch, Sputtering by Particle Bombardment (Springer, Berlin, 1981)

2. R. Behrisch, G. Betz, Sputtering by Particle Bombardment II: Sputtering of Alloys and Compounds (Springer, Berlin, 1983)

3. R. Behrisch, K. Wittmaack, Sputtering by Particle Bombardment III: Characteristics of Sputtered Particles, Technical Applications (Springer, Berlin, 1991)

4. R. Behrisch, W. Eckstein, Sputtering by Particle Bombardment: Experiments and Computer Calculations from Threshold to $\mathrm{MeV}$ Energies (Springer, Berlin, 2007)

5. W. Jacob, J. Roth, in Sputtering by Particle Bombardment, ed. by R. Behrisch, W. Eckstein (Springer, Berlin, 2007), pp. 329-400

6. I. Yamada, N. Toyoda, Nucl. Instrum. Methods B 241, 589 (2005) 
7. A. Delcorte et al., Anal. Chem. 79, 3673 (2007)

8. U. Helmersson, M. Lattemann, J. Bohlmark, A.P. Ehiasarian, J.T. Gudmundsson, Thin Solid Films 513, 1 (2006)

9. J.S. Colligon, Philos. Trans. R. Soc. Lond. A 362, 103 (2004)

10. T.L. Hu et al., J. Electron. Mater. 36, 81 (2007)

11. Y. Nunes et al., Vacuum 81, 1503 (2007)

12. S. Inoue, Y. Wada, K. Koterazawa, Vacuum 59, 735 (2000)

13. D. Herman, J. Sicha, J. Musil, Vacuum 81, 285 (2006)

14. S. Inoue, T. Namazu, H. Tawa, M. Niibe, K. Koterazawa, Vacuum 80, 744 (2006)

15. H. Hofsäss, C. Ronning, H. Feldermann, AIP Conf. Proc. 576, 947 (2001)

16. H. Hofsäss, H. Binder, T. Klumpp, E. Recknagel, Diam. Relat. Mater. 3, 137 (1994)

17. A. Anders, N. Pasaja, S. Sansongsiri, Rev. Sci. Instrum. 78, 063901 (2007)

18. P.B. Mirkarimi et al., J. Mater. Res. 9, 2925 (1994)

19. W. Kulisch, S. Ulrich, Thin Solid Films 423, 183 (2003)

20. F. Frost, R. Fechner, D. Flamm, B. Ziberi, W. Frank, A. Schindler, Appl. Phys. A 78, 651 (2004)

21. P.B. Mirkarimi et al., Microelectron. Eng. 77, 369 (2005)

22. W.L. Chan, E. Chason, J. Appl. Phys. 101, 121301 (2007)

23. U. Valbusa, C. Boragno, F. Buatier de Mongeot, Mater. Sci. Eng. C 23, 201 (2003)

24. J. Orloff, M. Utlaut, L. Swanson, High Resolution Focused Ion Beams: FIB and Its Applications (Kluver Academic/Plenum, New York, 2003)

25. B.A. McClure, D.C. New, U.S. Patent 6586816, 2000
26. K.L. Hobbs, P.R. Larson, G.D. Lian, J.C. Keay, M.B. Johnson, Nano Lett. 4, 167 (2004)

27. H. Huang, O.K. Tan, Y.C. Lee, M.S. Tse, J. Guo, T. White, Nanotechnology 17, 3668 (2006)

28. X.M. Meng, N.G. Shang, C.S. Lee, I. Bello, S.T. Lee, Phys. Status Solidi A 202, 2479 (2005)

29. J. Fu, J. van Gogh, U.S. Patent 6059945, 1998

30. S. Berg, I.V. Katardjiev, J. Vac. Sci. Technol. A 17, 1916 (1999)

31. S. Berg, I. Katardjiev, Surf. Coat. Technol. 84, 353 (1996)

32. S. Berg, A. Barklund, B. Gelin, C. Nender, I. Katardjiev, J. Vac. Sci. Technol. A 10, 1592 (1992)

33. J. Ziegler, J.P. Biersack, M.D. Ziegler, SRIM-The Stopping and Ranges of Ions in Solids (SRIM, Chester, 2008). http://srim.org

34. H. Hofsäss, K. Zhang, U.S. Patent pending, 2007

35. C.Y. Fong, M.D. Watson, L.H. Yang, S. Ciraci, Model. Simul. Mater. Sci. Eng. 10, R61-R77 (2002)

36. E. Tournié, K.H. Ploog, Thin Solid Films 231, 43-60 (1993)

37. S. Habenicht, W. Bolse, K.P. Lieb, Rev. Sci. Instrum. 69, 21202126 (1998)

38. K. Zhang et al., Surf. Coat. Technol. (2008, to be published); presented at SMMIB-15 conference, Mumbai, India, October 2007

39. Z. Pászti et al., Appl. Phys. A 76, 577-587 (2003)

40. W. Möller, W. Eckstein, J.P. Biersack, Comput. Phys. Commun. 51, 355 (1988)

41. K. Zhang et al., New. J. Phys. 9, 29 (2007)

42. H. Hofsäss, K. Zhang, J. Appl. Phys. 103, 083507 (2008) 